
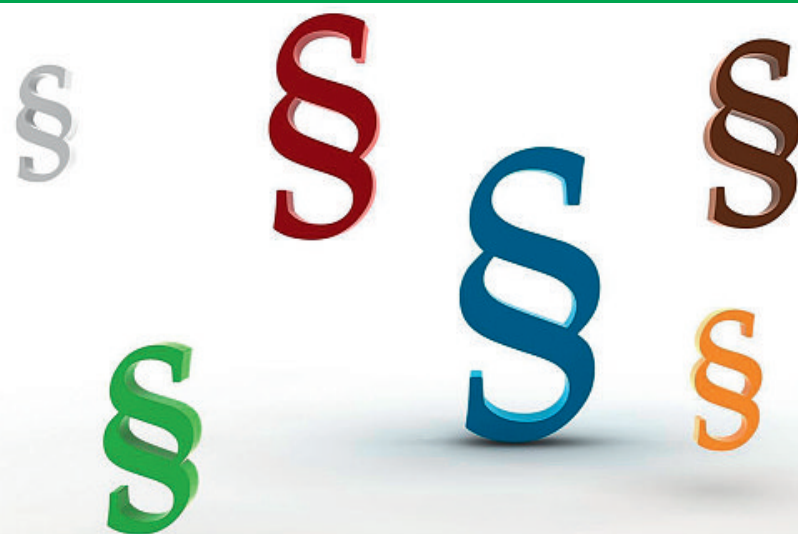

Rechtliche Aspekte im Umgang mit Minderjährigen

\section{Jugendliche in der Sprechstunde}

Brigitte Tag, Felicitas Witte

Korrespondenzen:

Prof. Dr. iur. utr. Brigitte Tag

Lehrstuhl für Strafrecht,

Strafprozessrecht

und Medizinrecht

Universität Zürich

Freiestrasse 15

CH-8032 Zürich

Tel. 0446343939

Fax 0446344937

Lst.Tag@rwi.uzh.ch

Dr. med. Felicitas Witte Rheinländerstrasse 12

CH-4056 Basel

Tel. 0613225830

Fax 0613225831

felicitas.witte@web.de

www.felicitas-witte.de
Ihre Mutter dürfe nicht wissen, dass sie schon mit ihrem Freund geschlafen habe. Sie sei noch zu jung, sich mit einem Mann einzulassen, habe die Mutter gesagt. Die 16-Jährige war zusammen mit ihrer Mutter in die gynäkologische Sprechstunde am Unispital Basel gekommen [1]. Als der Gynäkologe sie alleine ins Sprechzimmer bat, zögerte sie erst, war aber dann damit einverstanden. Alleine mit dem Arzt, erzählte sie ihm von schmerzhaften Regelblutungen - und von ihrem Freund, mit dem sie schlafen wollte. Eine Pille würde beide Probleme lösen. Doch darf der Arzt der Jugendlichen die Pille verschreiben? Und muss er der Mutter davon erzählen? «Ich möchte meine Tochter nicht kontrollieren, doch ich würde schon gerne wissen, wann und warum sie zum Arzt geht und sich z. B. die Pille verschreiben lässt», sagt Dorothea Engel, Mutter einer 13-Jährigen. «Aber sie bräuchte dafür nicht meine Erlaubnis. Als Eltern müssen wir akzeptieren, dass Kinder erwachsen werden und ihre eigenen Entscheidungen treffen. Auch Ärzte sollten Jugendliche als eigenständige Patienten respektieren.»

\section{Es gilt die Urteilsfähigkeit}

Grundlage der Behandlung Jugendlicher ist wie bei Erwachsenen der Behandlungsvertrag zwischen Arzt und Patient. Doch darf ein unmündiger Jugendlicher unter 18 Jahren einen Behandlungsvertrag abschliessen? Gemäss Zivilgesetzbuch dürfen auch urteilsfähige, aber noch unmündige Personen nur mit Zustimmung ihrer gesetzlichen Vertreter Verträge abschliessen. Es gibt hiervon jedoch einige Ausnahmen, zum Beispiel wenn urteilsfähige Minderjährige Rechte ausüben, die ihnen «um ihrer Persönlichkeit willen» zustehen. Der Abschluss eines Behandlungsvertrages gilt nach der Rechtssprechung als so ein «höchstpersönliches Recht». Urteilsfähige Jugendliche können also einen Behandlungsvertrag ohne Zustimmung ihrer Eltern oder des gesetzlichen Vertreters abschliessen, wenn es um eine alltägliche und nicht kostspielige Behandlung geht oder wenn die Kosten durch eine Krankenversicherung gedeckt sind.

Laut Gesetz gilt ein Mensch als urteilsfähig, der «nicht wegen seines Kindesalters oder infolge von Geisteskrankheit, Geistesschwäche, Trunkenheit oder

\section{Les adolescents en consultation}

Une jeune adolescente de 16 ans souhaite une ordonnance pour la pilule, mais ses parents ne doivent en aucun cas le savoir. Un jeune homme de 17 ans aimerait se faire enlever un fibrome qui l'affecte mais il ne veut pas attendre le retour de vacances de ses parents pour obtenir leur approbation avant d'opérer. Les adolescents disposent-ils de toute leur faculté de jugement, ont-ils le droit de décider euxmêmes d'un traitement médical et de donner leur approbation préalable? Evaluer le degré de discernement des jeunes représente un des enjeux des échanges avec les mineurs. Quand un adolescent estil apte à porter un jugement? La réponse à cette question ne dépend pas de l'âge de l'intéressé mais de sa maturité et d'une situation concrète. En soignant des jeunes, le médecin doit rester fidèle aux préceptes du secret médical, également face aux parents. Ne pas informer ces derniers d'un traitement ou d'une ordonnance pour la pilule peut être source de tension. Un dialogue ouvert et approfondi avec un adolescent au sujet du traitement qu'il souhaite et des éventuels conflits avec ses parents est synonyme d'une relation médecin/patient basée sur la confiance. En documentant clairement la faculté de jugement qu'il a pu observer chez un adolescent et les points abordés avec lui, le médecin dispose d'éléments qui pourront permettre d'éviter un malentendu ou un différend avec les parents.

ähnlichen Zuständen die Fähigkeit mangelt, vernunftgemäss zu handeln» (Art. 16 Zivilgesetzbuch; [2]). Der Jugendliche muss aufgrund seiner geistigen Reife in der Lage sein, den Zweck, die Wirkung und mögliche 
unerwünschte Wirkungen einer medizinischen oder medikamentösen Massnahme zu begreifen. Er muss verstehen, dass es möglicherweise alternative Behandlungsmöglichkeiten gibt und was passiert, wenn er eine Behandlung ablehnt. Bei dem 16-jährigen Mädchen am Unispital Basel bedeutete dies, ob sie über die verschiedenen Verhütungsmethoden Bescheid weiss, ob sie versteht, wie die Pille wirkt, wie sie sie

\section{Urteilsfähige Jugendliche haben ein Recht darauf, dass der Arzt niemandem von ihrem Gespräch berichtet. Hält er sich nicht daran, kann er bestraft werden}

einnehmen soll und was für Nebenwirkungen oder Probleme auftreten können, beispielsweise wenn sie einmal die Pille vergisst. Wann die Urteilsfähigkeit Jugendlicher beginnt, ist im Gesetz nicht definiert. Sie richtet sich nicht nach einer bestimmten Altersgrenze. Ob ein Jugendlicher urteilsfähig ist, muss der Arzt immer anhand der konkreten Situation prüfen und sollte dies auch dokumentieren. In der Regel geht man davon aus, dass Jugendliche ab einem Alter von etwa 13 bis 14 Jahren urteilsfähig sein können. Das ist jedoch individuell sehr unterschiedlich und hängt auch davon ab, welche Konsequenzen ein medizinischer Eingriff oder eine Medikamentenwirkung haben kann. So kann beispielsweise eine geistig weit entwickelte 13-Jährige in Bezug auf eine kleine medizinische Behandlung urteilsfähig sein, im Hinblick auf einen schwerwiegenden Eingriff mit grossen Risiken aber nicht. Manche Jugendliche können schon mit 13 vernünftige Entscheidungen treffen, andere erst mit 17.

«Damit ich einer jungen Frau die Pille verschreiben kann, schätze ich bereits im ersten Gespräch ihre Urteilsfähigkeit ein», sagt Metascha Stierli, niedergelassene Gynäkologin in Bern. «Ich glaube, einer der grössten Beweise der Urteilsfähigkeit einer jungen Frau in dieser Situation ist der Wunsch nach Verhütung.» Dieser Wunsch zeige, dass die Jugendliche wisse, dass sie schwanger werden könne. Viele Mädchen in so einer Situation suchten Informationen und

\footnotetext{
Hilfreiche Fragen, um die Urteilsfähigkeit einzuschätzen

- Ist der Jugendliche intellektuell und charakterlich seinem Lebensalter entsprechend entwickelt und gereift?

- Hat er die Informationen über die Diagnose, die vorgesehene Behandlung und die damit verbundenen Risiken verstanden?

- Ist er in der Lage, Vorteile und Risiken der Behandlung gegeneinander abzuwägen und allfällige Alternativen in Betracht zu ziehen?

- Kann er die Besonderheiten seiner individuellen Situation erkennen und sie seiner Entscheidung zugrunde legen?

- Weiss der Jugendliche, was er will, und kann er seinen eigenen Willen äussern?
}

hätten Fragen vorbereitet. «In Bezug auf eine mögliche Schwangerschaft handelt die Jugendliche so also <vernunftgemäss> und ich schätze sie als urteilsfähig ein», sagt Metascha Stierli. Beim zweiten Besuch bewertet die Gynäkologin die Urteilsfähigkeit erneut: «Ich versuche herauszufinden, ob sie weiterhin vernunftgemäss handelt, das heisst, ob sie die Pille korrekt einnimmt und ob Probleme aufgetreten sind.» Nähmen junge Mädchen die Pille nicht richtig ein, läge dies sehr selten daran, dass sie nicht urteilsfähig seien. «Viel häufiger sind Missverständnisse aufgetreten oder den Mädchen fehlten Informationen - das kläre ich im Gespräch.»

\section{Patientengeheimnis auch gegenüber den Eltern}

Vor einigen Jahren fragte der Pädiater Christoph Rutishauser vom Kinderspital Zürich 613 Jugendliche im Alter von 13 und 15 Jahren, was sie von einem Gespräch mit dem Arzt erwarteten [3]. Jeder dritte 13-Jährige und jeder zweite 15-Jährige wünschte sich, ab und zu den Arzt alleine zu sehen. Aber nur bei rund jedem fünften Jugendlichen kam es dazu. Um die Entwicklung des Jugendlichen zu einem eigenständigen Menschen zu unterstützen, gehört es dazu, ihn zumindest zu einem Teil des Gespräches alleine zu sehen. Manche Ärzte kündigen an, dass sie den Jugendlichen ab einem der nächsten Termine gerne alleine sehen würden. «Ich finde es absolut richtig, dass die Kinderärztin meine Tochter demnächst alleine untersucht», sagt Dorothea Engel. «Wichtig finde ich, dass der Arzt uns Eltern dies klar kommuniziert.»

Ein weiteres Ergebnis der Zürcher Umfrage war, dass 79\% der 13-Jährigen und 90\% der 15-Jährigen es wichtig finden, dass der Arzt das Patientengeheimnis wahrt. Doch nur knapp 40\% der Ärzte sprachen das Thema Patientengeheimnis mit den Jugendlichen an. Urteilsfähige Jugendliche haben ein Recht darauf, dass der Arzt niemandem von ihrem Gespräch berichtet auch nicht den Eltern. Hält sich der Arzt nicht daran, kann er bestraft werden (Art. 321 StGB, [4]). Im Gespräch mit dem Jugendlichen sollte der Arzt dem Jugendlichen das Patientengeheimnis erklären und ihm die Schweigepflicht zusichern. Beide vereinbaren, ob und welche Inhalte an die Eltern weitergegeben werden und was für Konsequenzen es haben kann, wenn man die Eltern nicht informiert.

So besprach der Basler Gynäkologe mit der 16-Jährigen unter vier Augen, ob er der Mutter im anschliessenden gemeinsamen Gespräch über das Pillenrezept berichten solle. Die Jugendliche war einverstanden aber der Arzt sollte nur erwähnen, dass sie die Pille gegen die Regelschmerzen bekäme und nicht zur Verhütung. «Mein erstes Ziel bei einer jugendlichen Patientin ist, ein Vertrauensverhältnis aufzubauen», sagt Gynäkologin Stierli. «Das erreiche ich am besten, wenn ich dem Mädchen erkläre, dass sie mir alle ihre Gedanken und Fragen offen legen darf und nicht befürchten muss, dass ich sie ihren Eltern weiterleite.» Eine 14-Jäh- 
rige kann sich also ohne Einwilligung ihrer Eltern die Pille oder andere Medikamente verschreiben lassen sofern sie urteilsfähig ist. Juristen empfehlen im Umgang mit Jugendlichen, das eingeschränkte Patientengeheimnis zuzusichern: Der Arzt vereinbart mit dem Jugendlichen, das Patientengeheimnis nicht zu wahren, wenn er den Eindruck hat, der Jugendliche könne sich selbst oder andere ernsthaft gefährden. einzuschätzen, ist einer der Kernpunkte im Gespräch mit einem jungen Patienten. Doch neben den rechtlichen Aspekten spielen auch die persönlichen Lebensumstände eine grosse Rolle: Würde ein Arzt beispielsweise auf Wunsch eines urteilsfähigen Jugendlichen eine Behandlung vornehmen, die die Eltern explizit ablehnen, könnte dies zu ziemlichen Unstimmigkeiten zwischen Jugendlichen und Eltern führen. Im aus-

\section{Weiss der Arzt vom älteren Freund und stellt trotzdem ein Pillenrezept aus, kann er dafür wegen Beihilfe zur sexuellen Handlung mit Unmündigen bestraft werden}

\section{Juristischer Zwiespalt}

Dieses Handeln kann aus strafrechtlicher Sicht problematisch werden, wenn ein Mädchen jünger als 16 Jahre alt ist und mit einem Mann schlafen möchte, der mehr als drei Jahre älter ist als sie. Hat zum Beispiel ein 21-Jähriger Sex mit einer 15-Jährigen, kann er dafür mit Freiheitsstrafe bis zu fünf Jahren oder einer Geldstrafe belangt werden. Die Parlamentarier legten den Altersunterschied auf drei Jahre fest, weil man davon ausgeht, dass bei einem grösseren Altersabstand die sexuelle Entwicklung der Jugendlichen gestört werden könnte. Möglicherweise könnte der oder die Ältere sexuelle Praktiken fordern, die das junge Mädchen oder der Bube noch nicht möchte. Weiss der Arzt nun von dem älteren Freund und stellt er trotzdem ein Pillenrezept aus, kann er dafür wegen Beihilfe zur sexuellen Handlung mit Unmündigen bestraft werden (Art. 187 StGB, [5]). Ein Arzt in einer solchen Situation befindet sich in einem grossen Zwiespalt: Einerseits darf er dem Mädchen die Pille nicht verschreiben, wenn er sich nicht selbst in strafrechtliche Probleme bringen will. Andererseits möchte er natürlich dafür sorgen, dass sie nicht schwanger wird. Einige Ärzte «befreien» sich aus diesem Dilemma, indem sie beispielsweise als Verschreibungsgrund «unreine Haut» oder «Regelschmerzen» auf das Rezept schreiben oder sie fragen das Mädchen nicht nach dem Alter ihres Freundes.

\section{Konflikt mit den Eltern}

Urteilsfähige Jugendliche haben das Recht, über eine medizinische Behandlung selbst zu entscheiden und ihre Einwilligung dazu zu geben. Die Urteilsfähigkeit führlichen Gespräch mit dem Jugendlichen sollte der Arzt deshalb auch einen möglichen Konflikt mit den Eltern ansprechen und dokumentieren. Manchmal ist ein Streit mit den Eltern notwendig, damit aus einem jungen Menschen ein Erwachsener mit einem eigenen Willen und einem eigenen Lebenskonzept wird. Doch der Arzt sollte nicht in diesen Konflikt mit einbezogen werden. Hier hilft eine klare Dokumentation: Der Arzt hat die Urteilsfähigkeit des jungen Patienten festgestellt und ihn ausführlich aufgeklärt.

\section{Literatur}

1 Bitzer J, Tschudin S, Frey Tirri B. Kontrazeption bei Jugendlichen. Schweiz Med Forum 2006;6:1004-10.

2 www.admin.ch/ch/d/sr/210/a16.html

3 Rutishauser C, Esslinger A, Bond L, Sennhauser F. Consultations with adolescents: the gap between their expectations and their experiences. Acta Paediatr 2003;92:1322-26.

4 www.admin.ch/ch/d/sr/311_0/a321.html

5 www.admin.ch/ch/d/sr/311_0/a187.html

\section{Weiterführende Literatur}

- Fellmann W. Arzt und das Rechtsverhältnis zum Patienten. In: Moritz Kuhn und Tomas Poledna (Hrsg.), Praxis des Arztrechts. Zürich: Schulthess; 2007. S. 103-231, insbesondere S. 115f.

- Tag B. Strafrecht im Arztalltag. In Kuhn/Poledna (ebd.). S. 669-765, insbesondere S. 669, 697ff. 